\title{
BIFURCATION ANALYSIS OF A SINGULAR ELLIPTIC PROBLEM MODELLING THE EQUILIBRIUM OF ANISOTROPIC CONTINUOUS MEDIA
}

\author{
Giovanni Molica Bisci — Vicenţiu D. Rădulescu
}

\begin{abstract}
In this work we obtain an existence result for a class of singular quasilinear elliptic Dirichlet problems on a smooth bounded domain containing the origin. By using a Caffarelli-Kohn-Nirenberg type inequality, a critical point result for differentiable functionals is exploited, in order to prove the existence of a precise open interval of positive eigenvalues for which the treated problem admits at least one nontrivial weak solution. In the case of terms with a sublinear growth near the origin, we deduce the existence of solutions for small positive values of the parameter. Moreover, the corresponding solutions have smaller and smaller energies as the parameter goes to zero.
\end{abstract}

\section{Introduction}

Stationary problems involving singular nonlinearities, as well as the associated evolution equations, describe naturally several physical phenomena. At our best knowledge, the first study in this direction is due to Fulks and Maybee [12], who proved existence and uniqueness results by using a fixed point argument; moreover, they showed that solutions of the parabolic problem tend to the unique solution of the corresponding stationary equation.

2010 Mathematics Subject Classification. Primary 35B32; Secondary 35J60, 58 E05.

Key words and phrases. Critical point theory, variational methods, Ricceri's variational principle, singular elliptic equations, bifurcation points.

This work is realized with with the support of Grants GNAMPA Project 2013: Problemi non-locali di tipo Laplaciano frazionario (G. Molina Bisci) and CNCS-PCCA-23/2014 (V. Rădulescu). 
Nonlinear singular boundary value problems arise in the context of chemical heterogeneous catalysts and chemical catalyst kinetics, in the theory of heat conduction in electrically conducting materials, singular minimal surfaces, as well as in the study of non-Newtonian fluids, boundary layer phenomena for viscous fluids; we refer for more details to [13], [14], [18], [19].

The problem we study in this paper corresponds to a model that describes several physical phenomena related to the equilibrium of anisotropic continuous media which possibly are somewhere "perfect" insulators or "perfect" conductors (see [11, p. 79]).

We are interested in the existence of solutions for the following singular elliptic Dirichlet problem

$$
\begin{cases}-\operatorname{div}\left(|x|^{-2 a} \nabla u\right)=\lambda|x|^{-2 b} f(u) & \text { in } \Omega, \\ u=0 & \text { on } \partial \Omega,\end{cases}
$$

where $\lambda$ is a real positive parameter and $\Omega$ is a bounded domain in $\mathbb{R}^{N}(N>2)$ with smooth boundary $\partial \Omega$ containing the origin, $a$ and $b$ are real numbers such that

$$
0 \leq a<\frac{N-2}{2} \text { and } a \leq b<a+1,
$$

and $f: \mathbb{R} \rightarrow \mathbb{R}$ is a continuous function satisfying the following subcritical growth condition

$$
\left(\mathrm{h}_{\infty}\right) \quad|f(t)| \leq a_{1}+a_{2}|t|^{r-1} \quad \text { for all } t \in \mathbb{R},
$$

for some nonnegative constants $a_{1}, a_{2}$ and $\left.r \in\right] 1, r_{\star}[$, where

$$
r_{\star}:=2 \min \left\{\frac{(N-2 b)}{N-2(a+1)}, \frac{N}{N-2}\right\} .
$$

If $r_{\star}=2 N /(N-2)$ some concentration phenomena may occur, due to the action of the non compact group of dilations in $\mathbb{R}^{N}$.

The most relevant feature in problem $\left(\mathrm{D}_{\lambda}^{a, b}\right)$ is due to the presence of the singular coefficients $|x|^{-2 a}$ and $|x|^{-2 b}$, which play a key role in the problem of existence of solutions when $0 \in \bar{\Omega}$.

In 1984, Caffarelli, Kohn and Nirenberg proved in [6], in the context of some more general inequalities, the following nice result:

Given $1<p<N$, there exists a positive constant $C_{a, b}$ such that, for every $u \in C_{0}^{\infty}(\Omega)$,

$$
\left(\int_{\Omega}|x|^{-b q}|u(x)|^{q} d x\right)^{p / q} \leq C_{a, b} \int_{\Omega}|x|^{-a p}|\nabla u(x)|^{p} d x,
$$

where

$$
-\infty<a<\frac{N-p}{p}, \quad a \leq b \leq a+1, \quad q=\frac{N p}{N-p(1+a-b)},
$$


and $\Omega \subseteq \mathbb{R}^{N}$ is an arbitrary open domain.

Note that the Caffarelli-Kohn-Nirenberg inequality (CKN) reduces to the classical Sobolev inequality (if $a=b=0$ ) and to the Hardy inequality (if $a=0$ and $b=1)$. Relation $(\mathrm{CKN})$ proves to be an important tool in studying degenerate elliptic problems. It is also related with the understanding of some important phenomena such as best constants, existence or nonexistence of extremal functions, symmetry properties of minimizers, compactness of minimizing sequences and concentration phenomena. We refer the reader to [3], [7]-[9], [15] and [17], [24], [27], [30], [31] for some relevant applications of (CKN); see also [26] for a Caffarelli-Kohn-Nirenberg type inequality with variable exponent and applications to PDE's.

In particular, in order to handle singular problems, various extensions of $(\mathrm{CKN})$ were achieved; see, for instance [1]. Moreover, in the last few years, existence, nonexistence and multiplicity of nonnegative solutions either for $\left(\mathrm{D}_{\lambda}^{a, b}\right)$ or some its variants, were obtained, where the nonlinear term is of pure power type; see [2], [20], [32], and references therein. In this direction, Ghergu and Rădulescu [16] studied the following nonlinear singular elliptic equation

$$
-\operatorname{div}\left(|x|^{-2 a} \nabla u\right)=K(x)|x|^{-b p}|u|^{p-2} u+\lambda g(x) \quad \text { in } \mathbb{R}^{N},
$$

where $g$ belongs to an appropriate weighted Sobolev space and $p$ denotes the Caffarelli-Kohn-Nirenberg critical exponent associated to the data $a, b$, and to the Euclidean dimension $N$. Under some natural assumptions on the positive potential $K(x)$, the existence of some $\lambda_{0}>0$ was proved such that the above problem has at least two distinct solutions provided that $\lambda \in\left(0, \lambda_{0}\right)$. The proof relies on Ekeland's variational principle and on the mountain pass theorem without the Palais-Smale condition, combined with a weighted variant of the celebrated Brezis-Lieb lemma (see [5] for the original version of the Brezis-Lieb lemma). More recently, Kristály studied problem $\left(\mathrm{D}_{\lambda}^{a, b}\right)$ and prove the existence of infinitely many distinct nonnegative solutions when $f$ is a continuous function oscillating either at the origin or at infinity; see [21, Theorems 1, 2].

Motivated by this large interest on the subject in the current literature, in this paper we exploit a quoted critical point result for differentiable functionals due to Ricceri (see Theorem 2.2 below) in order to prove the existence of a precise open interval of positive eigenvalues for which the treated problem admits at least one nontrivial weak solution; see Theorem 3.1 and Corollary 4.5. In addition, for $\lambda$ sufficiently small, the energy functional (namely $E_{\lambda}$ ) related to the problem is negative and decreasing on the solutions; see Remark 4.3.

We also observe that when the nonlinear term is sublinear at infinity, then the corresponding energy functional is coercive. In this case, the existence of a solution (possibly trivial) comes from standard arguments; see Remark 4.2 for 
details. It is worth noticing that, in our context, the potential may be also not coercive; see Example 4.6 and Remark 4.7.

We state here, as an example, the following special case of our results; see also Remark 4.4.

THEOREM 1.1. Let $0 \leq b<1$ and $f: \mathbb{R} \rightarrow \mathbb{R}$ be a continuous function such that

$$
\lim _{t \rightarrow 0^{+}} \frac{f(t)}{t}=+\infty, \quad \text { and } \quad \lim _{|t| \rightarrow \infty} \frac{f(t)}{|t|^{s}}=0,
$$

for some $0 \leq s<(N+2(1-2 b)) /(N-2)$. Then there exists $\lambda^{\star}>0$ such that for all $\lambda \in] 0, \lambda^{\star}[$, the following singular Dirichlet problem

$$
\begin{cases}-\Delta u=\lambda|x|^{-2 b} f(u) & \text { in } \Omega, \\ u=0 & \text { on } \partial \Omega,\end{cases}
$$

admits at least one nontrivial weak solution $u_{\lambda} \in H_{0}^{1}(\Omega)$. Moreover, we have

$$
\int_{\Omega}\left|\nabla u_{\lambda}(x)\right|^{2} d x \rightarrow 0, \quad \text { as } \lambda \rightarrow 0^{+}
$$

and the mapping

$$
\lambda \mapsto \frac{1}{2}\left(\int_{\Omega}\left|\nabla u_{\lambda}(x)\right|^{2} d x\right)-\lambda \int_{\Omega}|x|^{-2 b}\left(\int_{0}^{u_{\lambda}(x)} f(t) d t\right) d x,
$$

is negative and strictly decreasing in $] 0, \lambda^{\star}[$.

The paper is organized as follows. In Section 2, we recall some basic definitions and our main tool, while Section 3 is devoted to our abstract results. A concrete example is provided in the final section of thsi paper.

We refer to the monographs [16], [23], [25] for several related topics and a careful analysis of our abstract framework.

\section{Preliminaries}

Let $\Omega$ be a bounded domain in $\mathbb{R}^{N}$ with a smooth boundary $\partial \Omega$ and containing the origin. Denote by $\mathcal{D}_{a}^{1,2}(\Omega)$ the completion of $C_{0}^{\infty}(\Omega)$ with respect to the norm

$$
\|u\|_{a}=\left(\int_{\Omega}|x|^{-2 a}|\nabla u(x)|^{2} d x\right)^{1 / 2} .
$$

Then the embedding $j: \mathcal{D}_{a}^{1,2}(\Omega) \hookrightarrow L^{r}\left(\Omega ;|x|^{-q}\right)$ is continuous if

$$
1 \leq r \leq 2^{*}:=\frac{2 N}{N-2} \quad \text { and } \quad q \leq(1+a) r+N\left(1-\frac{r}{2}\right),
$$

where $L^{r}\left(\Omega ;|x|^{-q}\right)$ is the weighted Lebesgue space endowed with the norm

$$
\|u\|_{r, q}=\left(\int_{\Omega}|x|^{-q}|u(x)|^{r} d x\right)^{1 / r} .
$$


We refer to [10], [32] for more details.

Hence, there exists a positive constant $K_{r, q}^{a}$ such that

$$
\left(\int_{\Omega}|x|^{-q}|u(x)|^{r} d x\right)^{1 / r} \leq K_{r, q}^{a}\left(\int_{\Omega}|x|^{-2 a}|\nabla u(x)|^{2} d x\right)^{1 / 2},
$$

for every $u \in \mathcal{D}_{a}^{1,2}(\Omega)$. Moreover, the embedding $j$ is compact if

(C) $1 \leq r<2^{*}$ and $q<(1+a) r+N(1-r / 2)$.

Set

$$
F(\xi):=\int_{0}^{\xi} f(t) d t, \quad \text { for every } \xi \in \mathbb{R} .
$$

Consider the energy functional $E_{\lambda}: \mathcal{D}_{a}^{1,2}(\Omega) \rightarrow \mathbb{R}$ associated to $\left(\mathrm{D}_{\lambda}^{a, b}\right)$ defined as follows

$$
E_{\lambda}(u)=\Phi(u)-\lambda \Psi(u) \quad \text { for all } u \in \mathcal{D}_{a}^{1,2}(\Omega)
$$

where

$$
\Phi(u):=\frac{1}{2} \int_{\Omega}|x|^{-2 a}|\nabla u(x)|^{2} d x, \quad \Psi(u):=\int_{\Omega}|x|^{-2 b} F(u(x)) d x .
$$

Then $\Phi$ is a Gâteaux differentiable functional in $\mathcal{D}_{a}^{1,2}(\Omega)$ whose derivative is given by

$$
\Phi^{\prime}(u)(v)=\int_{\Omega}|x|^{-2 a} \nabla u(x) \cdot \nabla v(x) d x,
$$

for every $v \in \mathcal{D}_{a}^{1,2}(\Omega)$. Moreover, $\Phi$ is weakly lower semicontinuous and coercive.

On the other hand, we have the following preliminary result.

Lemma 2.1. Assume that $f$ verifies the growth condition $\left(\mathrm{h}_{\infty}\right)$. Then the functional $\Psi: \mathcal{D}_{a}^{1,2}(\Omega) \rightarrow \mathbb{R}$ is well defined, of class $C^{1}$, and sequentially weakly continuous.

Proof. The functional $\Psi$ is well defined since, by using (2.1) and exploiting (C), it follows that

$$
\Psi(u) \leq\left(a_{1} K_{1,2 b}^{a}+\frac{a_{2}}{r}\left(K_{r, 2 b}^{a}\right)^{r}\|u\|_{a}^{r-1}\right)\|u\|_{a}<+\infty,
$$

for every $u \in \mathcal{D}_{a}^{1,2}(\Omega)$.

Standard arguments show that $\Psi$ is a continuously Gâteaux differentiable functional whose Gâteaux derivative is

$$
\Psi^{\prime}(u)(v)=\int_{\Omega}|x|^{-2 b} f(u(x)) v(x) d x \quad \text { for every } v \in \mathcal{D}_{a}^{1,2}(\Omega) .
$$

Now, we prove that $\Psi$ is sequentially weakly continuous arguing by contradiction. Hence, let $\left\{u_{k}\right\} \subset \mathcal{D}_{a}^{1,2}(\Omega)$ be a sequence such that $u_{k} \rightarrow u$ in $\mathcal{D}_{a}^{1,2}(\Omega)$ but $\left\{\Psi\left(u_{k}\right)\right\}$ does not converges to $\Psi(u)$ as $n \rightarrow \infty$. Further, note that since

$$
r \in] 1, \min \left\{\frac{2(N-2 b)}{N-2(a+1)}, 2^{*}\right\}[,
$$


then $r$ and $2 b$ verify relation $(\mathrm{C})$. In other words

$$
1<r<2^{*} \text { and } 2 b<(1+a) r+N\left(1-\frac{r}{2}\right),
$$

see Remark 4.1 for details. Therefore, up to a subsequence, one can find a number $\varepsilon>0$ such that $0<\varepsilon \leq\left|\Psi\left(u_{k}\right)-\Psi(u)\right|$, for every $k \in \mathbb{N}$ and $u_{k} \rightarrow u$ in $L^{r}\left(\Omega ;|x|^{-2 b}\right)$. Moreover, for every $k \in \mathbb{N}$, we have $\left.\vartheta_{k} \in\right] 0,1[$ such that

$$
\begin{aligned}
\left|\Psi\left(u_{k}\right)-\Psi(u)\right| & \leq \int_{\Omega}|x|^{-2 b}\left|F\left(u_{k}(x)\right)-F(u(x))\right| d x \\
& =\int_{\Omega}|x|^{-2 b}\left|f\left(u(x)+\vartheta_{k}\left(u_{k}(x)-u(x)\right)\right)\right|\left|u_{k}(x)-u(x)\right| d x .
\end{aligned}
$$

Thus, from the above inequality and $\left(\mathrm{h}_{\infty}\right)$, it follows that

$$
\begin{aligned}
\left|\Psi\left(u_{k}\right)-\Psi(u)\right| \leq & a_{1} \int_{\Omega}|x|^{-2 b}\left|u_{k}(x)-u(x)\right| d x \\
& +a_{2} \int_{\Omega}|x|^{-2 b}\left|u(x)+\vartheta_{k}\left(u_{k}(x)-u(x)\right)\right|^{r-1}\left|u_{k}(x)-u(x)\right| d x .
\end{aligned}
$$

Next, we employ the Hölder inequality (with weight $|x|^{-2 b}$ ) and the following elementary property: for every real number $s>0$, then there exists a constant $c_{s}>0$ such that

$$
(\alpha+\beta)^{s} \leq c_{s}\left(\alpha^{s}+\beta^{s}\right) \text { for all } \alpha, \beta \in(0, \infty) .
$$

Hence (note that $r>1$ ) we can write

$$
\begin{aligned}
& \int_{\Omega}|x|^{-2 b}\left|u(x)+\vartheta_{k}\left(u_{k}(x)-u(x)\right)\right|^{r-1}\left|u_{k}(x)-u(x)\right| d x \\
& \left.\leq \int_{\Omega}|x|^{-2 b}\left(|u(x)|+\mid u_{k}(x)-u(x)\right) \mid\right)^{r-1}\left|u_{k}(x)-u(x)\right| d x \\
& =c_{r-1}\left(\int_{\Omega}|x|^{-2 b}|u(x)|^{r-1}\left|u_{k}(x)-u(x)\right| d x+\int_{\Omega}|x|^{-2 b}\left|u_{k}(x)-u(x)\right|^{r} d x\right) \\
& \leq c_{r-1}\left(\int_{\Omega}|x|^{-2 b}|u(x)|^{r} d x\right)^{(r-1) / r}\left(\int_{\Omega}|x|^{-2 b}\left|u_{k}(x)-u(x)\right|^{r} d x\right)^{1 / r} \\
& \quad+c_{r-1} \int_{\Omega}|x|^{-2 b}\left|u_{k}(x)-u(x)\right|^{r} d x \\
& =c_{r-1}\left(\|u\|_{r, 2 b}^{r-1}+\left\|u_{k}-u\right\|_{r, 2 b}^{r-1}\right)\left\|u_{k}-u\right\|_{r, 2 b .}
\end{aligned}
$$

Then

$$
\begin{aligned}
& 0<\varepsilon \leq\left|\Psi\left(u_{k}\right)-\Psi(u)\right| \\
& \quad \leq a_{1}\left\|u_{k}-u\right\|_{1,2 b}+c_{r-1} a_{2}\left(\|u\|_{r, 2 b}^{r-1}+\left\|u_{n}-u\right\|_{r, 2 b}^{r-1}\right)\left\|u_{k}-u\right\|_{r, 2 b} .
\end{aligned}
$$

Since $\left\{u_{k}\right\}$ strongly converges to $u$ in $L^{r}\left(\Omega ;|x|^{-2 b}\right)$ (so $u_{k} \rightarrow u$ in $L^{1}\left(\Omega ;|x|^{-2 b}\right)$ ), the right-hand side tends to zero as $k \rightarrow \infty$, contradicting $\varepsilon>0$. 
Fix $\lambda>0$. A function $u: \Omega \rightarrow \mathbb{R}$ is said to be a weak solution of $\left(\mathrm{D}_{\lambda}^{a, b}\right)$ if $u \in \mathcal{D}_{a}^{1,2}(\Omega)$ and

$$
\int_{\Omega}|x|^{-2 a} \nabla u(x) \cdot \nabla v(x) d x=\lambda \int_{\Omega}|x|^{-2 b} f(u(x)) v(x) d x \quad \text { for all } v \in \mathcal{D}_{a}^{1,2}(\Omega) .
$$

Hence, from the above remarks, the critical points of $E_{\lambda}$ are exactly the weak solutions of $\left(\mathrm{D}_{\lambda}^{a, b}\right)$.

Our main tool to prove the existence of a nontrivial weak solution for problem $\left(\mathrm{D}_{\lambda}^{a, b}\right)$ is the following critical point theorem due to Ricceri [28].

Theorem 2.2. Let $X$ be a reflexive real Banach space and $\Phi, \Psi: X \rightarrow \mathbb{R}$ be continuously Gâteaux differentiable functionals such that $\Phi$ is sequentially weakly lower semicontinuous and coercive and $\Psi$ is sequentially weakly upper semicontinuous. For every $\rho>\inf _{X} \Phi$, set

$$
\varphi(\rho):=\inf _{u \in \Phi^{-1}(]-\infty, \rho[)} \frac{\sup _{v \in \Phi^{-1}(]-\infty, \rho[)} \Psi(v)-\Psi(u)}{\rho-\Phi(u)} .
$$

Then, for every $\rho>\inf _{u \in X} \Phi(u)$ and every $\left.\lambda \in\right] 0,1 / \varphi(\rho)[$, the restriction of the functional $E_{\lambda}:=\Phi-\lambda \Psi$ to $\Phi^{-1}(]-\infty, \rho[)$ admits a global minimum, which is a critical point (local minimum) of $E_{\lambda}$ in $X$.

See also $[4$, Theorem 2.1, part (a)].

\section{Small energy solutions}

With the previous notations, the main abstract result of this paper reads as follows.

THEOREM 3.1. Let $f: \mathbb{R} \rightarrow \mathbb{R}$ be a continuous function satisfying condition $\left(\mathrm{h}_{\infty}\right)$. Assume that

$$
\limsup _{\xi \rightarrow 0^{+}} \frac{F(\xi)}{\xi^{2}}=+\infty \quad \text { and } \quad \liminf _{\xi \rightarrow 0^{+}} \frac{F(\xi)}{\xi^{2}}>-\infty .
$$

Then there exists a positive number $\lambda^{\star}$ given by

$$
\lambda^{\star}:=r \sup _{\gamma>0}\left(\frac{\gamma}{r a_{1} \sqrt{2} K_{1,2 b}^{a}+a_{2} 2^{r / 2}\left(K_{r, 2 b}^{a}\right)^{r} \gamma^{r-1}}\right),
$$

such that for every $\lambda \in] 0, \lambda^{\star}[$, the following elliptic Dirichlet problem

$$
\begin{cases}-\operatorname{div}\left(|x|^{-2 a} \nabla u\right)=\lambda|x|^{-2 b} f(u) & \text { in } \Omega, \\ u=0 & \text { on } \partial \Omega,\end{cases}
$$

admits at least one nontrivial weak solution $u_{\lambda} \in \mathcal{D}_{a}^{1,2}(\Omega)$. Moreover,

$$
\lim _{\lambda \rightarrow 0^{+}}\left\|u_{\lambda}\right\|_{a}=0 .
$$

and the function $\lambda \rightarrow E_{\lambda}\left(u_{\lambda}\right)$ is negative and strictly decreasing in $] 0, \lambda^{\star}[$. 
Proof. Fix $\lambda \in] 0, \lambda^{\star}\left[\right.$. Our aim is to apply Theorem 2.2 with $X:=\mathcal{D}_{a}^{1,2}(\Omega)$ and where $\Phi$ and $\Psi$ are the functionals introduced in Section 2. Note that $X$ is a Hilbert space whose inner product $\langle\cdot, \cdot\rangle: X \times X \rightarrow \mathbb{R}$ given by

$$
\langle u, v\rangle:=\int_{\Omega}|x|^{-2 a} \nabla u(x) \cdot \nabla v(x) d x \quad \text { for all } u, v \in X
$$

induces the norm $\|\cdot\|_{a}$. Hence $X$ is a reflexive space. Now, we prove that problem $\left(\mathrm{P}_{\lambda}\right)$ admits a weak solution $u_{\lambda} \in \Phi^{-1}(]-\infty, \bar{\rho}[)$, for a suitable $\bar{\rho}>0$.

For our goal observe that $\Phi: X \rightarrow \mathbb{R}$ is a continuously Gâteaux differentiable and sequentially weakly lower semicontinuous functional as well as the map $\Psi: X \rightarrow \mathbb{R}$ is continuously Gâteaux differentiable and sequentially weakly upper semicontinuous by Lemma 2.1. Moreover, $\Phi$ is coercive and $\inf _{u \in X} \Phi(u)=0$. Owing to $\left(\mathrm{h}_{\infty}\right)$, if follows that

$$
F(\xi) \leq a_{1}|\xi|+a_{2} \frac{|\xi|^{r}}{r} \quad \text { for all } \xi \in \mathbb{R} .
$$

Since $0<\lambda<\lambda^{\star}$, there exists $\bar{\gamma}>0$ such that

$$
\lambda<\frac{r \bar{\gamma}}{r a_{1} \sqrt{2} K_{1,2 b}^{a}+a_{2} 2^{r / 2}\left(K_{r, 2 b}^{a}\right)^{r} \bar{\gamma}^{r-1}}=: \lambda_{\bar{\gamma}}^{\star} .
$$

Now, set $\rho \in] 0,+\infty[$ and consider the function

$$
\chi(\rho):=\frac{\sup _{v \in \Phi^{-1}(]-\infty, \rho[)} \Psi(v)}{\rho} .
$$

Taking into account (3.1) it follows that

$$
\Psi(v):=\int_{\Omega}|x|^{-2 b} F(v(x)) d x \leq a_{1}\|v\|_{1,2 b}+\frac{a_{2}}{r}\|v\|_{r, 2 b}^{r} .
$$

Then, for every $v \in X$ such that $\Phi(v)<\rho$, we get

$$
\|v\|_{a}<\sqrt{2 \rho}, \quad \text { for every } v \in X .
$$

Now, from (2.1) and by using (3.3), for every $v \in X$ such that $\Phi(v)<\rho$, we obtain

Hence

$$
\Psi(v)<a_{1} K_{1,2 b}^{a} \sqrt{2 \rho}+\frac{a_{2} 2^{r / 2}\left(K_{r, 2 b}^{a}\right)^{r}}{r} \rho^{r / 2} .
$$

$$
\sup _{v \in \Phi^{-1}(]-\infty, \rho[)} \Psi(v) \leq a_{1} K_{1,2 b}^{a} \sqrt{2 \rho}+\frac{a_{2} 2^{r / 2}\left(K_{r, 2 b}^{a}\right)^{r}}{r} \rho^{r / 2} .
$$

Then

$$
\chi(\rho) \leq a_{1} K_{1,2 b}^{a} \sqrt{\frac{2}{\rho}}+\frac{a_{2} 2^{r / 2}\left(K_{r, 2 b}^{a}\right)^{r}}{r} \rho^{r / 2-1},
$$

for every $\rho>0$. In particular, we deduce that

$$
\chi\left(\bar{\gamma}^{2}\right) \leq \frac{a_{1} \sqrt{2} K_{1,2 b}^{a}}{\bar{\gamma}}+\frac{a_{2} 2^{r / 2}\left(K_{r, 2 b}^{a}\right)^{r}}{r} \bar{\gamma}^{r-2} .
$$


At this point, observe that

$$
\varphi\left(\bar{\gamma}^{2}\right):=\inf _{u \in \Phi^{-1}(]-\infty, \bar{\gamma}^{2}[)} \frac{\sup _{v \in \Phi^{-1}(]-\infty, \bar{\gamma}^{2}[)} \Psi(v)-\Psi(u)}{\bar{\gamma}^{2}-\Phi(u)} \leq \chi\left(\bar{\gamma}^{2}\right),
$$

taking into account that $0_{X} \in \Phi^{-1}(]-\infty, \bar{\gamma}^{2}[)$ and $\Phi\left(0_{X}\right)=\Psi\left(0_{X}\right)=0$.

In conclusion, bearing in mind (3.2), the above inequality together with (3.5) yield

$$
\varphi\left(\bar{\gamma}^{2}\right) \leq \chi\left(\bar{\gamma}^{2}\right) \leq \frac{a_{1} \sqrt{2} K_{1,2 b}^{a}}{\bar{\gamma}}+\frac{a_{2} 2^{r / 2}\left(K_{r, 2 b}^{a}\right)^{r}}{r} \bar{\gamma}^{r-2}<\frac{1}{\lambda} .
$$

In other words,

$$
\lambda \in] 0, \frac{r \bar{\gamma}}{r a_{1} \sqrt{2} K_{1,2 b}^{a}+a_{2} 2^{r / 2}\left(K_{r, 2 b}^{a}\right)^{r} \bar{\gamma}^{r-1}}[\subseteq] 0,1 / \varphi\left(\bar{\gamma}^{2}\right)[.
$$

Thanks to Theorem 2.2, there exists a function $u_{\lambda} \in \Phi^{-1}(]-\infty, \bar{\gamma}^{2}[)$ such that $E_{\lambda}^{\prime}\left(u_{\lambda}\right)=\Phi^{\prime}\left(u_{\lambda}\right)-\lambda \Psi^{\prime}\left(u_{\lambda}\right)=0$, and, in particular, $u_{\lambda}$ is a global minimum of the restriction of $E_{\lambda}$ to $\Phi^{-1}(]-\infty, \bar{\gamma}^{2}[)$.

Next, we prove that the function $u_{\lambda}$ cannot be trivial $\left(u_{\lambda} \neq 0_{X}\right)$. To this end, let us prove that

$$
\limsup _{\|u\| \rightarrow 0} \frac{\Psi(u)}{\Phi(u)}=\limsup _{\|u\| \rightarrow 0} \frac{\int_{\Omega}|x|^{-2 b} F(u(x)) d x}{\|u\|_{a}^{2} / 2}=+\infty .
$$

Thanks to our assumptions at zero, we can fix a sequence $\left\{\xi_{k}\right\} \subset \mathbb{R}^{+}$converging to zero and two constant $\sigma$, and $\kappa$ (with $\sigma>0$ ) such that, for every $\xi \in[0, \sigma]$,

$$
\lim _{k \rightarrow \infty} \frac{\int_{0}^{\xi_{k}} f(t) d t}{\xi_{k}^{2}}=+\infty \text { and } \int_{0}^{\xi} f(t) d t \geq \kappa \xi^{2} .
$$

Now, let us consider a closed ball $\bar{B}\left(x_{0}, \tau\right) \subset \Omega$ (not containing zero) for some $x_{0} \in \Omega$ and $\tau>0$ sufficiently small. Further, let $v \in C_{0}^{\infty}(\Omega) \subset X$ such that

(a) $v(x) \in[0,1]$, for every $x \in \Omega$;

(b) $v(x)=1$, for every $x \in \bar{B}\left(x_{0}, \tau / 2\right)$;

(c) $v(x)=0$, for every $x \in \Omega \backslash B\left(x_{0}, \tau\right)$.

Finally, fix $\varepsilon>0$ and consider a real positive number $\mu$ with

$$
\varepsilon<2 \frac{\mu \int_{B\left(x_{0}, \tau / 2\right)}|x|^{-2 b} d x+\kappa \int_{C}|x|^{-2 b} v(x)^{2} d x}{\|v\|_{a}^{2}},
$$

where we set $C:=B\left(x_{0}, \tau\right) \backslash B\left(x_{0}, \tau / 2\right)$. Note that the right-hand side of the above inequality is finite. Indeed since $0 \notin B\left(x_{0}, \tau / 2\right)$, then

$$
\int_{B\left(x_{0}, \tau / 2\right)}|x|^{-2 b} d x<+\infty \text {. }
$$


Moreover, since the injection $j: X \hookrightarrow L^{2}\left(\Omega ;|x|^{-2 b}\right)$ is continuous, taking into account that $1<2<2^{*}$ and $2 b<2(a+1)$, we deduce that there exists a constant $K_{2,2 b}^{a}>0$ (according to our notations) such that

$\int_{C}|x|^{-2 b} v(x)^{2} d x<\int_{\Omega}|x|^{-2 b} v(x)^{2} d x \leq\left(K_{2,2 b}^{a}\right)^{2}\left(\int_{\Omega}|x|^{-2 a}|\nabla u(x)|^{2} d x\right)<+\infty$.

Then, there is $\nu \in \mathbb{N}$ such that $\xi_{k}<\delta$ and

$$
\int_{0}^{\xi_{k}} f(t) d t \geq \mu \xi_{k}^{2}, \quad \text { for every } k>\nu .
$$

At this point, for every $k>\nu$, and bearing in mind the properties of the function $v$, we obtain

$$
\begin{aligned}
\frac{\Psi\left(\xi_{k} v\right)}{\Phi\left(\xi_{k} v\right)} & =2 \frac{\int_{B\left(x_{0}, \tau / 2\right)}|x|^{-2 b}\left(\int_{0}^{\xi_{k}} f(t) d t\right) d x+\int_{C}|x|^{-2 b} F\left(\xi_{k} v(x)\right) d x}{\left\|\xi_{k} v\right\|_{a}^{2}} \\
& \geq 2 \frac{\mu \int_{B\left(x_{0}, \tau / 2\right)}|x|^{-2 b} d x+\kappa \int_{C}|x|^{-2 b} v(x)^{2} d x}{\|v\|_{a}^{2}}>\varepsilon .
\end{aligned}
$$

Since $\varepsilon$ can be chosen arbitrarily large, it follows that

$$
\lim _{k \rightarrow \infty} \frac{\Psi\left(\xi_{k} v\right)}{\Phi\left(\xi_{k} v\right)}=+\infty
$$

from which (3.6) clearly follows.

Hence, there exists a sequence $\left\{w_{k}\right\} \subset X$ strongly converging to zero, such that for every $k$ sufficiently large, $w_{k} \in \Phi^{-1}(]-\infty, \bar{\gamma}^{2}[)$, and

$$
E\left(w_{k}\right):=\Phi\left(w_{k}\right)-\lambda \Psi\left(w_{k}\right)<0 .
$$

Since $u_{\lambda}$ is a global minimum of the restriction of $E_{\lambda}$ to $\Phi^{-1}(]-\infty, \bar{\gamma}^{2}[)$, we conclude that

$$
\Phi\left(u_{\lambda}\right)-\lambda \Psi\left(u_{\lambda}\right)<0=E_{\lambda}\left(0_{X}\right)
$$

hence $u_{\lambda}$ is not trivial.

Now, we prove that $\lim _{\lambda \rightarrow 0^{+}}\left\|u_{\lambda}\right\|_{a}=0$. Bearing in mind that $\Phi$ is a coercive functional and that $u_{\lambda} \in \Phi^{-1}(]-\infty, \bar{\gamma}^{2}[)$, for every $\left.\lambda \in\right] 0, \lambda \lambda_{\gamma}^{\star}$, we obtain

$$
\left\|u_{\lambda}\right\|_{a}<\sqrt{2} \bar{\gamma}
$$

Therefore, from $\left(\mathrm{h}_{\infty}\right)$, taking into account (2.1) and the above inequality, it follows that there exists $M_{\bar{\gamma}}>0$ such that

$$
\left.\left|\int_{\Omega}\right| x\right|^{-2 b} f\left(u_{\lambda}(x)\right) u_{\lambda}(x) d x \mid \leq M_{\bar{\gamma}},
$$

for every $\lambda \in] 0, \lambda_{\bar{\gamma}}^{\star}[$. 
Now, $E_{\lambda}^{\prime}\left(u_{\lambda}\right)=0$, for every $\left.\lambda \in\right] 0, \lambda_{\bar{\gamma}}^{\star}\left[\right.$ and in particular $E_{\lambda}^{\prime}\left(u_{\lambda}\right)\left(u_{\lambda}\right)=0$, that is,

$$
\left\|u_{\lambda}\right\|_{a}^{2}=\lambda \int_{\Omega}|x|^{-2 b} f\left(u_{\lambda}(x)\right) u_{\lambda}(x) d x
$$

for every $\lambda \in] 0, \lambda_{\gamma}^{\star}[$. Then, from (3.7), it follows that

$$
\lim _{\lambda \rightarrow 0^{+}}\left\|u_{\lambda}\right\|_{a}^{2}=\lim _{\lambda \rightarrow 0^{+}} \lambda \Psi^{\prime}\left(u_{\lambda}\right)\left(u_{\lambda}\right)=0 .
$$

Hence, as claimed, $\lim _{\lambda \rightarrow 0^{+}}\left\|u_{\lambda}\right\|_{a}=0$.

Next, we observe that

$$
E_{\lambda}(u)=\lambda\left(\frac{\Phi(u)}{\lambda}-\Psi(u)\right),
$$

for every $u \in X$. Fix $0<\lambda_{1}<\lambda_{2}$ (with $\lambda_{2}<\lambda_{\bar{\gamma}}^{\star}$ ). Set

$$
m_{\lambda_{1}}:=\left(\frac{\Phi\left(u_{\lambda_{1}}\right)}{\lambda_{1}}-\Psi\left(u_{\lambda_{1}}\right)\right)=\inf _{u \in \Phi^{-1}(] 0, \bar{\gamma}^{2}[)}\left(\frac{\Phi(u)}{\lambda_{1}}-\Psi(u)\right),
$$

and

$$
m_{\lambda_{2}}:=\left(\frac{\Phi\left(u_{\lambda_{2}}\right)}{\lambda_{2}}-\Psi\left(u_{\lambda_{2}}\right)\right)=\inf _{u \in \Phi^{-1}(] 0, \bar{\gamma}^{2}[)}\left(\frac{\Phi(u)}{\lambda_{2}}-\Psi(u)\right) .
$$

Clearly, as claimed before, $m_{\lambda_{i}}<0$ (for $i=1,2$ ), and $m_{\lambda_{2}} \leq m_{\lambda_{1}}$ thanks to $\lambda_{1}<\lambda_{2}$. Then the mapping $\lambda \mapsto E_{\lambda}\left(u_{\lambda}\right)$ is strictly decreasing in $] 0, \lambda^{\star}$ [ owing to

$$
E_{\lambda_{2}}\left(u_{\lambda_{2}}\right)=\lambda_{2} m_{\lambda_{2}} \leq \lambda_{2} m_{\lambda_{1}}<\lambda_{1} m_{\lambda_{1}}=E_{\lambda_{1}}\left(u_{\lambda_{1}}\right) .
$$

This concludes the proof.

\section{Additional properties and examples}

In this section we provide several related results and examples.

REMARK 4.1. By simple direct computations, since

$$
0 \leq a<\frac{N-2}{2} \text { and } a \leq b<a+1,
$$

it follows that

$$
2<\frac{2(N-2 b)}{N-2(a+1)} .
$$

Further, we observe that if, instead of $0 \leq a<(N-2) / 2$, we require the more restrictive condition $0 \leq a<b(N-2) / N$, one has

$$
\left.\frac{2(N-2 b)}{N-2(a+1)} \in\right] 2,2^{*}[\text {. }
$$

Thus, in this special case, the above relation yields

$$
r_{\star}=\frac{2(N-2 b)}{N-2(a+1)} .
$$


On the other hand if $a=b$, since

$$
\frac{N-2 a}{N-2(a+1)} \geq \frac{N}{N-2},
$$

it follows that $r_{\star}=2^{*}$. Finally, we point out that the parameter $\lambda^{\star}$ in Theorem 3.1 has the following expression

$$
\lambda^{\star}= \begin{cases}+\infty & \text { if } 1<r<2, \\ \frac{1}{a_{2}\left(K_{2,2 b}^{a}\right)^{2}} \quad r \widetilde{\gamma}_{\max } & \text { if } r=2, \\ \frac{r a_{1} \sqrt{2} K_{1,2 b}^{a}+a_{2} 2^{r / 2}\left(K_{r, 2 b}^{a}\right)^{r} \widetilde{\gamma}_{\max }^{r-1}}{} & \text { if } r \in] 2, r_{\star}[,\end{cases}
$$

where

$$
\widetilde{\gamma}_{\max }:=\frac{1}{\sqrt{2}}\left(\frac{a_{1} r K_{1,2 b}^{a}}{a_{2}\left(K_{r, 2 b}^{a}\right)^{r}(r-2)}\right)^{1 /(r-1)} .
$$

REMARK 4.2. From the above expressions, it follows that if $f$ is sublinear at infinity, Theorem 3.1 ensures that for all $\lambda>0$, the problem $\left(\mathrm{D}_{\lambda}^{a, b}\right)$ admits at least one nontrivial weak solution. We explicitly observe that in this case, also the classical direct methods theorem (Tonelli's result) ensures the existence of at least one weak solution that anyway can be trivial.

REMARK 4.3. We observe that if $f(0)=0$, Theorem 3.1 is a bifurcation result. Indeed, in this setting, it follows that the trivial solution solves problem $\left(\mathrm{D}_{\lambda}^{a, b}\right)$ for every parameter $\lambda$. Hence, $\lambda=0$ is a bifurcation point for problem $\left(\mathrm{D}_{\lambda}^{a, b}\right)$, in the sense that the point $(0,0)$ belongs to the closure of the set

$$
\mathfrak{F}:=\left\{(u, \lambda) \in \mathcal{D}_{a}^{1,2}(\Omega) \times\right] 0,+\infty\left[: u \text { is a weak solution of }\left(\mathrm{D}_{\lambda}^{a, b}\right), u \neq 0\right\}
$$

in the space $\mathcal{D}_{a}^{1,2}(\Omega) \times \mathbb{R}$. Otherwise, if $f(0) \neq 0$ evidently one has

$$
\lim _{\xi \rightarrow 0^{+}} \frac{F(\xi)}{\xi^{2}}=+\infty \text {. }
$$

Hence, all the assumptions of Theorem 3.1 are automatically verified and the conclusions follow.

REMARK 4.4. Theorem 1.1 in Introduction easily follows from Theorem 3.1 taking into account that the following $s$-sublinear assumption at infinity

$$
\lim _{|t| \rightarrow \infty} \frac{f(t)}{|t|^{s}}=0
$$

in which $s \in\left[0, s_{\star}\right)$, where

$$
s_{\star}:=\min \left\{\frac{N+2(a-2 b+1)}{N-2(a+1)}, \frac{N+2}{N-2}\right\},
$$

implies the growth condition $\left(\mathrm{h}_{\infty}\right)$. In addition, if

$$
\lim _{t \rightarrow 0^{+}} \frac{f(t)}{t}=+\infty,
$$


also condition $\left(\mathrm{h}_{0}^{\prime}\right)$ holds true. Finally, bearing in mind Remark 4.1, the parameter $\lambda^{\star}$ has the form

$$
\lambda^{\star}= \begin{cases}+\infty & \text { if } 0 \leq s<1, \\ \frac{1}{\bar{a}_{2}\left(K_{2,2 b}^{a}\right)^{2}} & \text { if } s=1, \\ \frac{s-1}{2 K_{1,2 b}^{a} s}\left(\frac{\bar{a}_{1}(s+1) K_{1,2 b}^{a}}{\bar{a}_{2}\left(K_{s+1,2 b}^{a}\right)^{s+1}(s-1)}\right)^{1 / s} & \text { if } s \in] 1, s_{\star}[,\end{cases}
$$

for suitable nonnegative real constants $\bar{a}_{1}, \bar{a}_{2}$.

The next result is an immediate consequence of Remark 4.1.

COROLlary 4.5. Let $f: \mathbb{R} \rightarrow \mathbb{R}$ be a continuous function satisfying the following subcritical growth condition

$$
|f(t)| \leq a_{1}+a_{2}|t|^{r-1} \quad \text { for all } t \in \mathbb{R},
$$

where $a_{1}, a_{2}$ are nonnegative constants and $\left.r \in\right] 2, r_{\star}[$. Further, assume that condition $\left(\mathrm{h}_{0}^{\prime}\right)$ holds. Then, for every

$$
\lambda \in] 0, \frac{r-2}{2 K_{1,2 b}^{a}(r-1)}\left(\frac{a_{1} r K_{1,2 b}^{a}}{a_{2}\left(K_{r, 2 b}^{a}\right)^{r}(r-2)}\right)^{1 /(r-1)}[,
$$

problem $\left(\mathrm{D}_{\lambda}^{a, b}\right)$ admits at least one nontrivial weak solution $u_{\lambda} \in \mathcal{D}_{a}^{1,2}(\Omega)$. Moreover,

$$
\lim _{\lambda \rightarrow 0^{+}}\left\|u_{\lambda}\right\|_{a}=0 .
$$

and the function $\lambda \rightarrow E_{\lambda}\left(u_{\lambda}\right)$ is negative and strictly decreasing in $] 0, \lambda^{\star}[$.

The next example deals with a nonlinearity $f$ that vanishes at zero. The existence of one nontrivial solution for the Dirichlet problem involving the map $f$ is achieved by using Corollary 4.5 .

EXAMPLE 4.6. Let $\Omega$ be a bounded open subset of $\mathbb{R}^{N}$ (containing the origin) with $N \geq 3$, and consider the following problem

$$
\left(\widetilde{\mathrm{D}}_{\lambda}^{a, b}\right) \quad \begin{cases}-\operatorname{div}\left(|x|^{-2 a} \nabla u\right)=\lambda|x|^{-2 b} f(u) & \text { in } \Omega, \\ u=0 & \text { on } \partial \Omega,\end{cases}
$$

where $f(u):=|u|^{h-2} u+|u|^{s-2} u, 1<h<2$ and $2<s<r_{\star}$. Then, for every

$$
\lambda \in] 0, \frac{s-2}{2 K_{1,2 b}^{a}(s-1)}\left(\frac{s K_{1,2 b}^{a}}{\left(K_{s, 2 b}^{a}\right)^{s}(s-2)}\right)^{1 /(s-1)}[,
$$

problem $\left(\widetilde{\mathrm{D}}_{\lambda}^{a, b}\right)$ admits at least one nontrivial weak solution $u_{\lambda} \in \mathcal{D}_{a}^{1,2}(\Omega)$ such that

$$
\lim _{\lambda \rightarrow 0^{+}}\left\|u_{\lambda}\right\|_{a}=0 .
$$


To prove this, we can apply Corollary 3.1 with

$$
f(t):=|t|^{h-2} t+|t|^{s-2} t,
$$

for every $t \in \mathbb{R}$. In fact, $f(0)=0$ and it is easy to verify that

$$
|f(t)| \leq 2\left(1+|t|^{s-1}\right) \quad \text { for all } t \in \mathbb{R} .
$$

Then, condition $\left(\mathrm{h}_{\infty}^{\prime}\right)$ holds. Next, a direct computation shows that

$$
\lim _{\xi \rightarrow 0^{+}} \frac{F(\xi)}{\xi^{2}} \geq \frac{1}{h}\left(\lim _{\xi \rightarrow 0^{+}} \frac{1}{\xi^{2-h}}\right)=+\infty .
$$

Hence, assumption $\left(\mathrm{h}_{0}^{\prime}\right)$ is verified and the conclusion follows.

REMARK 4.7. We point out that the energy functional $E_{\lambda}$ associated to problem $\left(\widetilde{\mathrm{D}}_{\lambda}^{a, b}\right)$ is unbounded from below. Hence, since the functional $E_{\lambda}$ is not coercive, the classical direct method result cannot be applied to the case treated in Example 4.6.

REMARK 4.8. In conclusion, we also note that a related bifurcation result (in respect to Theorem 3.1) for perturbed nonsingular elliptic Dirichlet problems and involving the classical Laplace operator $\Delta u:=\operatorname{div}(\nabla u)$ was successfully studied by Ricceri; see [29, Theorem 1] for completeness. On the contrary of the cited contribution, in Theorem 3.1 we find a concrete expression of the parameter $\lambda^{\star}$ also in the laplacian case without singularities $(a=b=0)$. More precisely, if $f$ is subcritical (hence $\left(\mathrm{h}_{\infty}\right)$ holds), under our assumptions at zero, problem $\left(\mathrm{D}_{\lambda}^{0,0}\right)$ admits at least one nontrivial weak solution $u_{0, \lambda} \in H_{0}^{1}(\Omega)$ for every $\left.\lambda \in\right] 0, \lambda^{\star}[$, in which

$$
\lambda^{\star}:= \begin{cases}+\infty & \text { if } 1<r<2, \\ \frac{1}{a_{2} c_{2}^{2}} & \text { if } r=2, \\ \frac{r-2}{2 c_{1}(r-1)}\left(\frac{r a_{1} c_{1}}{a_{2} c_{r}^{r}(r-2)}\right)^{1 /(r-1)} & \text { if } r \in] 2,2^{*}[,\end{cases}
$$

where the numbers $c_{r}\left(r \in\left[2,2^{*}[)\right.\right.$ are the best Sobolev constants of the continuous inclusion $H_{0}^{1}(\Omega) \hookrightarrow L^{r}(\Omega)$. Moreover, also in this case, we have

$$
\int_{\Omega}\left|\nabla u_{0, \lambda}(x)\right|^{2} d x \rightarrow 0
$$

as $\lambda \rightarrow 0^{+}$and the mapping

$$
\lambda \mapsto \frac{1}{2}\left(\int_{\Omega}\left|\nabla u_{0, \lambda}(x)\right|^{2} d x\right)-\lambda \int_{\Omega}\left(\int_{0}^{u_{0, \lambda}(x)} f(t) d t\right) d x,
$$

is negative and strictly decreasing in $] 0, \lambda^{\star}[$. 


\section{REFERENCES}

[1] B. Abdellaoui, E. Colorado and I. Peral, Some improved Caffarelli-Kohn-Nirenberg inequalities, Calc. Var. Partial Differential Equations 23 (2005), 327-345.

[2] B. Abdellaoui and I. Peral, Existence and nonexistence results for quasilinear elliptic equations involving the p-Laplacian with a critical potential, Ann. Mat. Pura Appl. 182 (2003), 247-270.

[3] M. Badiale and G. Tarantello, A Sobolev-Hardy inequality with applications to a nonlinear elliptic equation arising in astrophysics, Arch. Ration. Mech. Anal. 163 (2002), 259-293.

[4] G. Bonanno and G. Molica Bisci, Infinitely many solutions for a Dirichlet problem involving the p-Laplacian, Proc. Roy. Soc. Edinburgh Sect. A 140 (2009), 1-16.

[5] H. Brezis And E. Lieb, A relation between pointwise convergence of functions and convergence of functionals, Proc. Amer. Math. Soc. 88 (1983), 486-490.

[6] L. Caffarelli, R. Kohn and L. Nirenberg, First order interpolation inequalities with weights, Compositio Math. 53 (1984), 259-275.

[7] P. Caldiroli and R. Musina, On the existence of extremal functions for a weighted Sobolev embedding with critical exponent, Calc. Var. Partial Differential Equations 8 (1999), 365-387.

[8] _ On a variational degenerate elliptic problem, Nonlinear Differential Equations Appl. (NoDEA) 7 (2000), 187-199.

[9] F. Catrina And Z.-Q. WANG, On the Caffarelli-Kohn-Nirenberg inequalities: sharp constants, existence (and nonexistence) and symmetry of extremal function, Commun. Pure Appl. Math. 54 (2001), 229-258.

[10] K.S. Chou And C.W. Chu, On the best constant for a weighted Sobolev-Hardy inequality, J. London Math. Soc. 48 (1993), 137-151.

[11] R. Dautray and J.-L. Lions, Mathematical Analysis and Numerical Methods for Science and Technology, Vol. 1, Physical Origins and Classical Methods, Springer-Verlag, Berlin, 1985.

[12] W. Fulks and J.S. Maybee, A singular nonlinear equation, Osaka J. Math. 12 (1960), $1-19$.

[13] M. Ghergu And V. Rădulescu, On a class of sublinear singular elliptic problems with convection term, J. Math. Anal. Appl. 311 (2005), 635-646.

[14] _ Multiparameter bifurcation and asymptotics for the singular Lane-EmdenFowler equation with a convection term, Proc. Roy. Soc. Edinburgh Sect. A 135 (2005), $61-84$.

[15] _ Singular elliptic problems with lack of compactness, Ann. Mat. Pura Appl. 185 (2006), 63-79.

[16] _ Singular Elliptic Problems: Bifurcation and Asymptotic Analysis, Oxford Lecture Series in Mathematics and its Applications, No. 37, Oxford University Press, New York, 2008.

[17] N. Ghoussoub And C. YUAn, Multiple solutions for quasi-linear PDEs involving the critical Sobolev and Hardy exponents, Trans. Amer. Math. Soc. 352 (2000), 5703-5743.

[18] Y. HAITAO, Multiplicity and asymptotic behavior of positive solutions for a singular semilinear elliptic problem, J. Differential Equations 189 (2003), 487-512.

[19] J. Hernández, F.J. Mancebo and J.M. Vega, On the linearization of some singular nonlinear elliptic problems and applications, Ann. Inst. H. Poincaré Anal. Non Linéaire 19 (2002), 777-813. 
[20] L. ItURriaga, Existence and multiplicity results for some quasilinear elliptic equation with weights, J. Math. Anal. Appl. 339 (2008), 1084-1102.

[21] A. KRIStÁLy, On singular elliptic equations involving oscillatory terms, Nonlinear Anal. 72 (2010), 1561-1569.

[22] A. KRistály And Cs. VARga, Multiple solutions for elliptic problems with singular and sublinear potentials, Proc. Amer. Math. Soc. 135 (2007), 2121-2126.

[23] A. Kristály, V. RĂdulescu And Cs. VArga, Variational Principles in Mathematical Physics, Geometry, and Economics: Qualitative Analysis of Nonlinear Equations and Unilateral Problems, Encyclopedia of Mathematics and its Applications, No. 136, Cambridge University Press, Cambridge, 2010.

[24] E.H. Lieb, Sharp constants in the Hardy-Littlewood-Sobolev and related inequalities, Ann. Math. 118 (1983), 349-374.

[25] J. Mawhin and M. Willem, Critical Point Theory and Hamiltonian Systems, Applied Mathematical Sciences, vol. 74, Springer-Verlag, New York, 1989.

[26] M. MihăIlescu, V. RĂdulescu And D. Stancu, A Caffarelli-Kohn-Nirenberg type inequality with variable exponent and applications to PDE's, Complex Var. Elliptic Equ. 56 (2011), 659-669.

[27] P. PuCCI AND R. ServadeI, Existence, non-existence and regularity of radial ground states for p-Laplacian equations with singular weights, Ann. Inst. H. Poincaré Anal. Non Linéaire 25 (2008), 505-537.

[28] B. RICCERI, A general variational principle and some of its applications, J. Comput. Appl. Math. 113 (2000), 401-410.

[29] _ A bifurcation theory for some nonlinear elliptic equations, Colloq. Math. 95 (2003), 139-151.

[30] D. Smets And M. Willem, Partial symmetry and asymptotic behavior for some elliptic variational problems, Calc. Var. Partial Differential Equations 18 (2003), 57-75.

[31] Z.-Q. Wang And M. Willem, Singular minimization problems, J. Differential Equations 161 (2000), 307-320.

[32] B. XUAN, The solvability of quasilinear Brezis-Nirenberg type problems with singular weights, Nonlinear Anal. 62 (2005), 703-725.

G. Molica BiscI

Dipartimento Patrimonio

Architettura e Urbanistica

University of Reggio Calabria

Feo di Vito

89124 Reggio Calabria, ITALY

E-mail address: gmolica@@unirc.it

V. RĂDULESCU

Department of Mathematics

Faculty of Science

King Abdulaziz University

Jeddah, SAUDI ARABIA

E-mail address: vicentiu.radulescu@@math.cnrs.fr

TMNA : Volume $45-2015-\mathrm{N}^{\mathrm{O}} 2$ 